\title{
Gallbladder Papillary Neoplasm with Intermediate Grade Intraepithelial Neoplasia
}

National Cancer Institute

\section{Source}

National Cancer Institute. Gallbladder Papillary Neoplasm with Intermediate Grade

Intraepithelial Neoplasia. NCI Thesaurus. Code C96878.

A non-invasive, papillary epithelial neoplasm that arises from the epithelium of

gallbladder. It is characterized by the presence of moderate epithelial atypia. 